\title{
Morphological evidence that activated polymorphs circulate in the peripheral blood of patients with rheumatoid arthritis
}

School of

Biological Sciences,

Queen Mary and

Westfield College, London, UK D A McCarthy

J Bernhagen

Immunology

Department,

St. Thomas' Hospital, London, UK

M J Taylor

A S Hamblin

Rheumatology

Department,

The London Hospital,

London, UK

I James

P W Thompson

J D Perry

Correspondence to:

Dr D A McCarthy,

School of Biological Sciences,

Queen Mary and

Wile End Road,

London El 4NS, UK.

Accepted for publication

12 February 1991

\author{
D A McCarthy, J Bernhagen, M J Taylor, A S Hamblin, I James, P W Thompson, J D Perry
}

\begin{abstract}
Purified peripheral blood polymorphonuclear leucocytes (PMNs) from patients with rheumatoid arthritis (RA) have been found to differ from purified PMNs from normal subjects in ways that are consistent with their prior activation. However, it is currently contentious whether activated PMNs really circulate in patients with RA, or whether they are produced as an in vitro artefact of purification. Recently developed rapid leucocyte fixation and preparation technique showed that the proportion of polarised (activated) PMNs (36.9 (24.7)\%, mean (SD); $n=31$ ) was increased relative to that in control subjects $(8 \cdot 1(5 \cdot 6) \% ; n=12)$. Serum cytidine deaminase levels, a biochemical marker of PMN lysis, were also increased in patients with RA (11.59 (7.26) $\mathrm{U} / \mathrm{ml}$ ) compared with those in controls $(6.82$ (3.78) $\mathrm{U} / \mathrm{ml}$ ), but the proportion of polarised PMNs and the levels of cytidine deaminase activity were unrelated to clinical assessments of inflammatory disease activity.
\end{abstract}

Twelve patients who were not receiving drugs or who were receiving only non-steroidal anti-inflammatory drugs (NSAIDs) had more polarised PMNs than 19 patients receiving second line treatment in addition to NSAIDs (patients receiving NSAIDs, 49.6 (25.9)\%; patients receiving second line treatment, 27.5 (21.1)\%). Fluorescence activated cytometric analysis of CR1 and CR3 expression on PMNs from a randomly selected subgroup of patients with RA showed that the serum level of cytidine deaminase activity was correlated positively with the expression of CRI (the C3b receptor) on the cell surface and that the proportion of polarised PMNs was positively correlated with the expression of CR3 (or CD11b/CD18), the iC3b receptor that is upregulated on activation. It is suggested that the polarised PMNs which circulate in blood samples from patients with RA represent cells which have been activated but not yet marginated, or activated cells which have marginated but subsequently returned to the circulating pool.

Preparations of peripheral blood polymorphonuclear leucocytes (PMNs) from patients with rheumatoid arthritis (RA) have frequently been found to contain cells which show evidence of prior activation. ${ }^{12}$ For example, preparations of PMNs from patients with RA contain more cells with a low electrophoretic mobility ${ }^{3}$ and a polarised (non-spherical) shape $e^{4}$ than do similar preparations from control subjects. It is surpris- ing that substantial numbers of activated PMNs can be recovered from patients with $R A$ by venepuncture, because if normal PMNs are activated in vitro, they show an increased adherence to cultured endothelial cells $s^{5}$ and the infusion of activators, for example, C5a, into animals in vivo causes a transient neutropenia that is attributed to increased margination. ${ }^{6}$ It is possible that the differences seen between purified PMNs from patients with RA and control subjects might possibly be an artefact due to selective changes in vitro during the purification procedure, as blood samples from patients with RA often contain substances such as immune complexes for example, that are capable of eliciting similar changes in normal PMNs, for example, decreases in electrophoretic mobility.

One way to resolve this issue might be by immediately fixing PMNs in blood samples ex vivo and subsequent examination. A convenient indicator of activation in this respect is cell shape, as it has been shown that the polarising responses in PMNs that are elicited by chemotactic agents in vitro can be easily monitored by light microscopy. ${ }^{8}$ Moreover, a rapid leucocyte fixation and preparation technique has recently been developed specifically for this purpose. ${ }^{9}$ We have used this technique to look for morphological evidence of PMN activation in blood samples of patients with RA and control subjects. In addition, we have assessed the activity of cytidine deaminase (CD) in serum samples and have also examined the surface expression of CR1 and CR3 on PMNs in subgroups of patients and controls, as these parameters might be expected to correlate with the extent of PMN activation.

\section{Patients and methods}

PATIENTS AND CONTROLS

Thirty one patients ( 22 women, nine men, mean age 56 years, range 28-81 years) who had classical or definite RA (American Rheumatism Association criteria) were studied. Inflammatory disease activity was assessed on a scale of $0-3$ $(0=$ none, $3=$ maximum $)$ by a combination of the duration of early morning stiffness, joint activity, an articular index, laboratory parameters where available (erythrocyte sedimentation rate, $\mathrm{C}$ reactive protein, white blood cell count) and a pain score. Two patients were classified as grade 0,14 as grade 1 , nine as grade 2 and six as grade 3. Four patients were receiving no drugs and all of the others were receiving NSAIDs. In addition, eight were receiving penicillamine, four cytotoxic drugs, five steroids and cytotoxic drugs, three steroids 
and penicillamine, one penicillamine and a cytotoxic drug, one gold salts and one chloroquine. Twenty two patients were latex positive; 12 had extra-articular disease.

The control subjects were healthy individuals (four women, eight men, mean age 37 years, range 21-55 years) who were receiving no drugs and who had no known inflammatory conditions.

All patients and controls gave their informed consent for the study.

\section{LEUCOCYTE PREPARATION AND SHAPE ANALYSIS OF PMNS}

The leucocytes were prepared for light microscopy as described previously. ${ }^{9}$ Briefly, $1 \mathrm{ml}$ venous blood drawn from the median antecubital vein was immediately mixed with $1 \mathrm{ml}$ pre-warmed $\left(37^{\circ} \mathrm{C}\right)$ phosphate buffered saline (PBS) or Hanks's buffered salt solution with $0.01 \mathrm{M}$ Hepes pH 7.3 buffer (HHBSS) containing $0.4 \%$ formaldehyde and kept for $4 \mathrm{~min}$ at $37^{\circ} \mathrm{C}$. Erythrocytes were lysed by the addition of $40 \mathrm{ml} 0 \cdot 155 \mathrm{M}$ ammonium chloride $\mathrm{pH} 7 \cdot 3$ (or in some instances $0.155 \mathrm{M}$ ammonium chloride buffered with $0.01 \mathrm{M}$ Tris chloride or $0.01 \mathrm{M}$ Hepes, pH 7.3). Leucocytes were collected by centrifuging at $200 \mathrm{~g}$ for $5 \mathrm{~min}$, washed twice by centrifuging at $200 \mathrm{~g}$ for $10 \mathrm{~min}$ in PBS or HHBSS $(40 \mathrm{ml}$ and $20 \mathrm{ml}$ consecutively), resuspended in $1 \mathrm{ml}$ PBS or HHBSS and stored at $0^{\circ} \mathrm{C}$. Preparations were mixed ( $1: 1)$ with toluidine blue $(0.01 \%$ in PBS) and the cells allowed to settle by gravity onto poly-L-lysine coated cover slips. When the cells had adhered, the cover slip was placed inverted on a cavity slide and examined using a Leitz microscope with bright field illumination and an oil immersion $(\times 70)$ objective lens. Photomicrographs were taken with a Leitz Dialux 20 microscope.

Neutrophils were assigned by one of three observers (who, whenever possible, were blind to the diagnosis) into one of the following categories on the basis of shape: spherical, slightly polar, moderately polar or strongly polar. ${ }^{9}$ At least 150 well separated cells were counted in each preparation and the percentage score was calculated for each category. Altogether a total of 17 leucocyte preparations were made on different occasions from the 12 control subjects (five from one subject, two from another, and one each from the remainder), and one preparation from each of the patients with RA. Whenever practicable, a blood sample from a control subject was processed alongside a blood sample from one or more patients with RA.

\section{DETERMINATION OF SURFACE EXPRESSION OF} CR1 AND CR3

Samples $(25 \mu \mathrm{l})$ of the leucocyte preparations from a randomly selected subgroup of the patients with RA $(n=14)$ and control subjects $(n=12)$ were labelled within two hours with monoclonal antibodies kindly supplied by Professor P C Beverley, Professor W Bodmer, Dr N Hogg and Professor A McMichael, which were either ascites fluid that had been titrated to give a working dilution (usually 1:1000) or undiluted culture supernatants (table). Incubations were performed on a microtitre plate (Falcon 3911 Microtest III, Becton Dickinson, Cowley, Oxford, UK) at $0^{\circ} \mathrm{C}$ for 30 minutes and the cells were washed after each incubation with $100 \mu \mathrm{l}$ of medium. RPMI medium containing $5 \%$ fetal calf serum was used throughout. Controls cells were prepared which were either incubated with mouse monoclonal antibody alone or with a subclass matched irrelevant monoclonal antibody (UCHTI). Fluorescein conjugated rabbit anti-mouse $\left(\mathrm{Fc} \mathrm{F}(\mathrm{ab})_{2}\right)$ diluted 1:50 (Dakopatts, Weybridge, Surrey, UK) was used as the second layer. Labelled cells were resuspended in $25 \mu$ l of RPMI fetal calf serum, transferred to round bottomed polystyrene tubes containing $1 \%$ paraformaldehyde, then stored for two hours in the dark at $40^{\circ} \mathrm{C}$ and analysed by flow cytometry using a FACscan (Becton Dickinson, Mountain View, CA, USA) equipped with Consort 30 software (Hewlett Packard). The polymorphs were separated on the basis of their forward and side scatter; their identity was verified by staining with monoclonal antibodies which identify leucocytes (W6/32 and 2D1) and leucocyte subpopulations (29 and TG1). Specificity was verified with a subclass matched control antibody (UCHT1). Fluorescence data were collected on a four decade logarithmic scale; markers were set on the control antibody (UCHT1). There was generally less than $5 \%$ non-specific staining. The mean fluorescence intensity ratio was calculated by dividing the mean fluorescence intensity of the polymorph population labelled with a given monoclonal antibody by the mean fluorescence intensity of the same population labelled with the negative control antibody. These experiments formed part of a larger study of leucocyte integrin and CR 1 surface expression

Monoclonal antibodies used in indirect fluorescence cytometry

\begin{tabular}{|c|c|c|c|c|}
\hline $\begin{array}{l}\text { Monoclonal } \\
\text { antibody }\end{array}$ & $\begin{array}{l}C D \\
\text { antigen }\end{array}$ & Cell specificity & Isotype & Reference \\
\hline $\begin{array}{l}\text { W6/32a } \\
2 \mathrm{D} 1^{a} \\
29^{b} \\
\text { TG1 }^{b} \\
\text { UCHTI }^{c} \\
\text { MHM23 } \\
44^{b} \\
\mathrm{E}^{b} 3^{b}\end{array}$ & $\begin{array}{l}- \\
\text { CD45 } \\
\text { CD15 } \\
\text { Unknown } \\
\text { CD3 } \\
\text { CD18 } \\
\text { CD11b } \\
\text { CD35 }\end{array}$ & $\begin{array}{l}\text { All leucocytes (HLA,A,B,C) } \\
\text { All leucocytes } \\
\text { Polymorphs, monocytes, natural killer cells } \\
\text { Polymorphs, monocytes } \\
\text { T lymphocytes } \\
\text { All leucocytes } \\
\text { Polymorphs, monocytes, natural killer cells (CR3) } \\
\text { Polymorphs, monocytes } \\
\text { Lymphocytes (CR1) }\end{array}$ & $\begin{array}{l}\text { IgG1 } \\
\text { IgG1 } \\
\text { IgM } \\
\text { IgM } \\
\text { IgGI } \\
\text { IgG1 } \\
\text { IgG1 } \\
\text { IgG1 }\end{array}$ & $\begin{array}{l}10 \\
11 \\
12 \\
12 \\
13 \\
14 \\
15 \\
16\end{array}$ \\
\hline
\end{tabular}

a Supplied by Professor W Bodmer.

h Supplied by Dr N Hogg.

c Supplied by Professor P C Beverley.

d Supplied by Professor A McMichael. 
in patients with RA and controls which will be presented elsewhere.

MEASUREMENT OF CD ACTIVITY

A portion $(4 \mathrm{ml})$ of the blood collected by venepuncture was allowed to clot at $37^{\circ} \mathrm{C}$ for one hour. Serum was separated by centrifugation, frozen within two hours and kept at $-20^{\circ} \mathrm{C}$ until assayed. The $C D$ activity was estimated in samples obtained from all but one of the patients with RA and all but one of the controls, essentially as described previously, ${ }^{17}$ except that the samples were incubated with cytidine at $37^{\circ} \mathrm{C}$ for four hours. This modification yields estimates of $C D$ activity which are routinely 3.25 times higher than those published previously. ${ }^{17}$

STATISTICAL ANALYSIS

Differences between the subject groups were

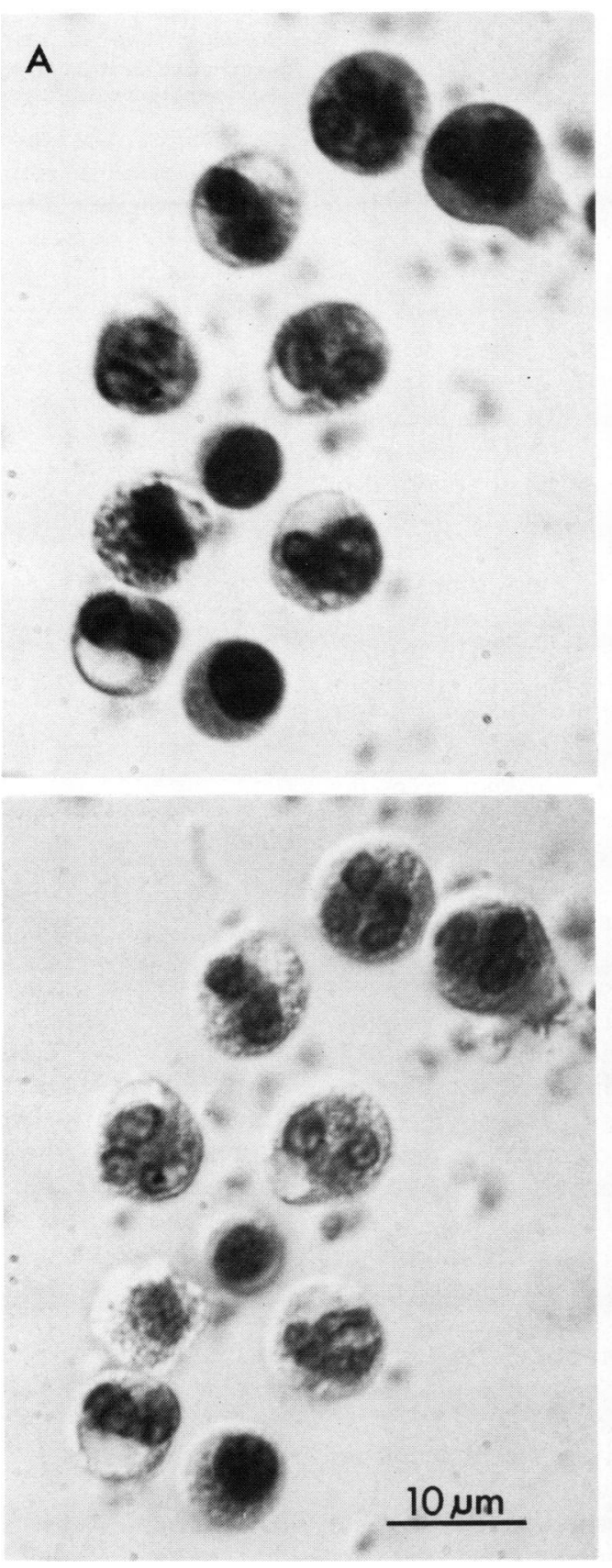

compared by the Mann-Whitney test with twotailed $\mathbf{p}$ values. Correlations were made using Spearman's rank correlation test and Pearson's linear regression analysis. $p$ Values less than 0.05 were considered significant when comparisons were being made between groups and values of less than 0.01 were considered significant when multiple correlations were being tested.

\section{Results}

In the leucocyte preparations from control subjects, most of the neutrophils were spherical or slightly polarised and the proportions of moderately and strongly polarised cells were always low. Compared with control subjects. the percentages of spherical cells were decreased and those of slightly polarised, moderately polarised and strongly polarised cells were

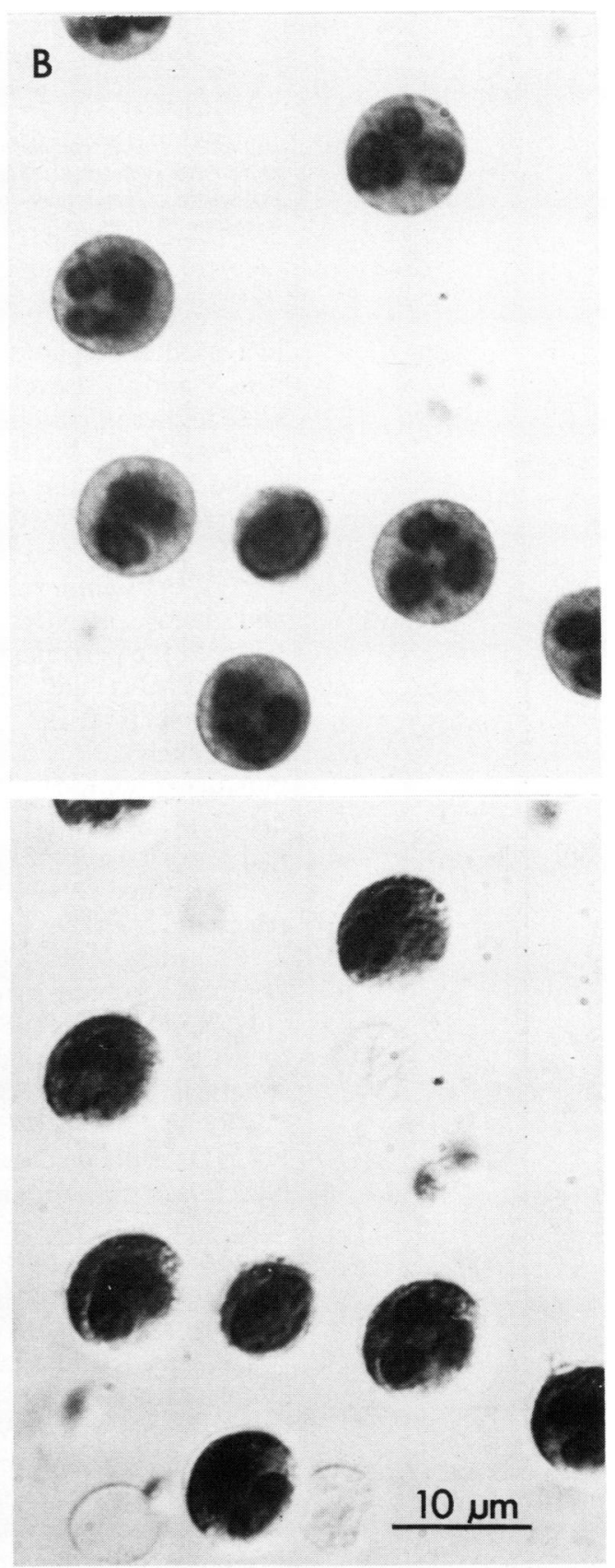

Figure 1 Toluidine blue stained leucocyte preparations from $(A)$ a patient with $R A$ and $(B)$ a control subject. Upper panel: bright field illumination. Lower panel: Normarski differential interference contrast. 


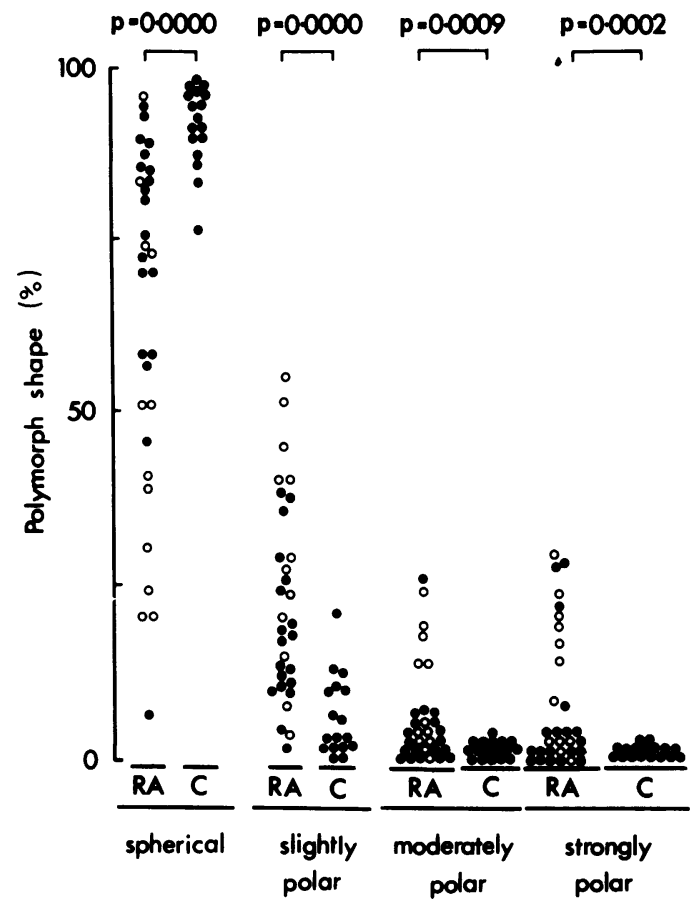

Figure 2 Polymorph morphology in patients with $R A$ and control subjects $(C)$. Shape categories are as described previously. ${ }^{9}$ Open symbols represent those patients with $R A$ who were receiving only NSAIDs or no drugs.

increased in preparations from patients with RA (figs 1 and 2). Levels of $C D$ in serum samples were higher in patients with RA than in control subjects (fig 3). No significant correlations were found between the percentages of spherical or strongly polar PMNs and disease activity $(\mathrm{r}=$ $0 \cdot 108, p=0.60$ and $r=0.08, p=0.68$, respectively), between levels of $\mathrm{CD}$ in serum samples and disease activity, $(r=0.026, p=0.900)$, or between the percentages of spherical or strongly polar PMNs and levels of $C D$ in serum $(r=0.244, p=0.193$, and $r=0.192, p=0.308$, respectively).

Patients with RA who were not receiving drugs, or who were receiving only NSAIDs, had fewer $(p=0.037)$ spherical PMNs (median $45 \cdot 6 \%$, range $20 \cdot 4-95 \cdot 8 \%, n=12$ ) than those receiving NSAIDs and other drug(s) (median $75 \cdot 0 \%$, range 6.9-94.6\%, $n=19$ ). Patients who were not receiving drugs or who were receiving only NSAIDS, or NSAIDs with choloroquine or with penicillamine, had fewer $(p=0.015)$ spherical PMNs (median $51.0 \%$, range $6.9-95.8 \%, n=17$ ) than those receiving NSAIDs with gold salts or cytotoxic drugs or NSAIDs with combinations of steroids, penicillamine and cytotoxic drugs (median $77.8 \%$, range $57 \cdot 0-94 \cdot 6 \%, n=14$ ). No correlations were found between levels of $C D$ in serum samples and drug treatment.

The mean values of the mean fluorescence intensity ratios for CR1 and CR3 on PMNs from patients with RA (mean (2SD) $1.76(0.29)$ and $3.70(0.62)$, respectively) were increased slightly compared with control PMNs (1.71 $(0.34)$ and $2.88(0.67)$, respectively), but these differences were not considered significant $(p=$ 0.682 for CR1 and $p=0.085$ for CR3). Linear and rank correlation analysis showed that for

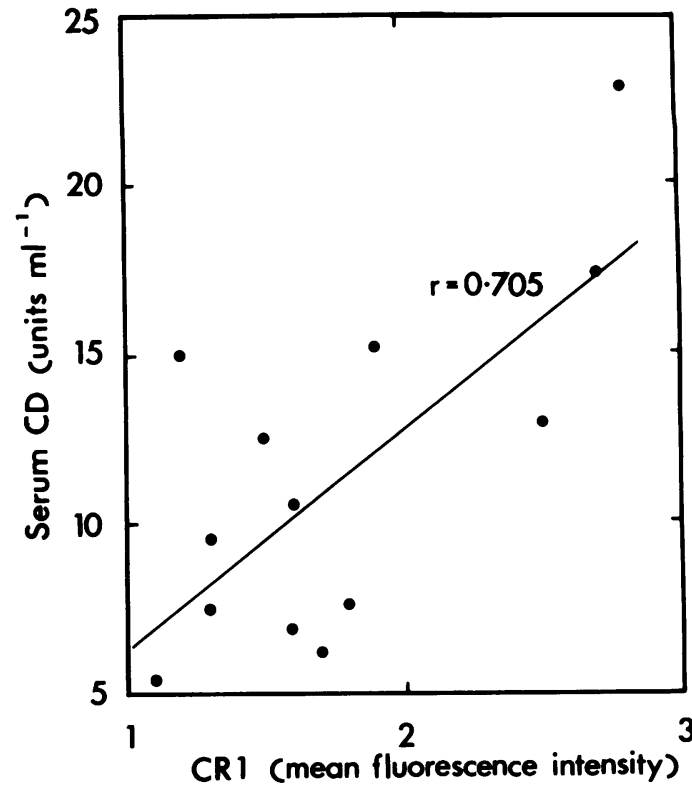

Figure 4 Correlation between the levels of serum $C D$ activity and the mean fluorescence intensity ratio of $C R I$ on polymorphs from patients with $R A$.

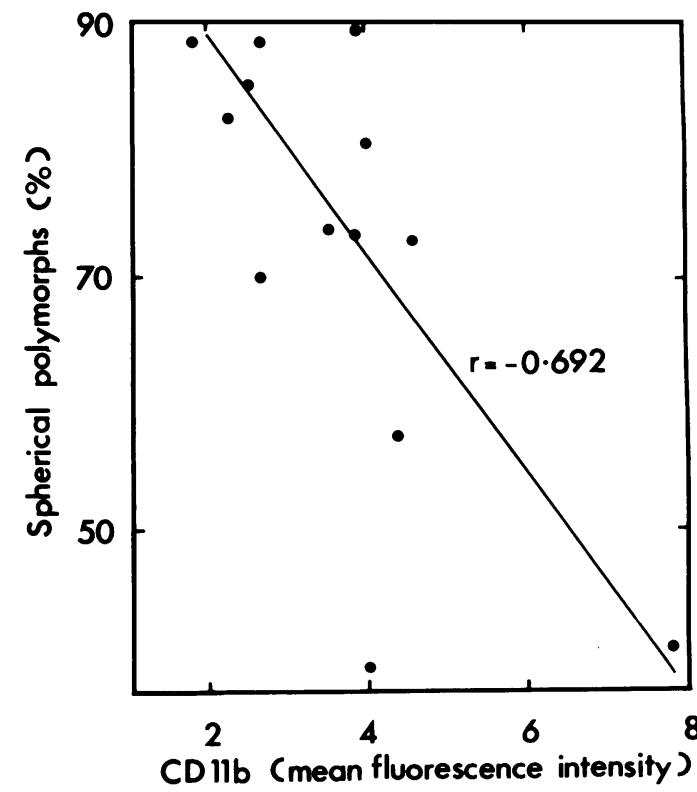

Figure 5 Correlation between the percentage of spherical polymorphs and the mean fluorescence intensity ratio of $C R 3$ on polymorphs in patients with $R A$.

PMNs from patients with RA, the mean fluorescence intensity ratio of $C R 1$ was positively correlated with the activity of $C D$ in serum samples $(r=0.705, p=0.007 ; \varrho=0.590, p=0.01)$ (fig 4) and that the mean fluorescence intensity ratio of CR3 was negatively correlated with the percentage of spherical cells $(r=-0.692$, $p=0.009 ; \rho=-0.575, p<0.025$ ) (fig 5). Also for this subgroup of patients, the disease activity was weakly correlated with $C D$ activity in $\operatorname{serum}(r=0.597, p=0.031 ; \varrho=0.461, p<0.1)$.

\section{Discussion}

Except for the unlikely possibility that changes occurred selectively in blood samples from patients with RA during venepuncture, our 
results provide unequivocal evidence that the morphology of peripheral blood PMNs from patients with RA differs from that of PMNs from control subjects. Moreover, the range of morphology seen among PMNs from patients with RA resembles that seen in normal PMNs when blood samples have been incubated with chemotaxins in vitro. ${ }^{9}$ As polarisation is just one of many responses which PMNs make when activated by chemotaxins in vitro,,$^{18} 19$ the simplest explanation of these results is that the polarised cells seen in blood samples from patients with RA represent a subpopulation of cells which have been activated by chemotaxins in vivo. In addition, there is ample evidence for the increased production of activated complement components and inflammatory cytokines in patients with RA. ${ }^{20-22}$

Substantial increases in the surface expression of CR1 and CR3 occur rapidly when PMNs are exposed to chemotaxins in vitro, ${ }^{23} 24$ but only insignificant increases were found on PMNs from patients with RA. Although this result does not support the contention that chemotaxin activated PMNs can be found in the circulating leucocyte pool of patients with RA, it should be remembered that the concentration of chemotaxins required to elicit secretory functions in PMNs is an order of magnitude higher than that needed to elicit chemotactic functions. For instance, the concentration of formyl methionyl-leucylphenylalanine required for half-maximum increases in CR3 expression is $1.25-2.5 \times 10^{-9}$ $\mathrm{mol} / 1,{ }^{23}$ whereas concentrations of $7 \times 10^{-11}$ and $1 \times 10^{-10} \mathrm{~mol} / \mathrm{l}$ elicit half-maximal responses in chemotactic migration ${ }^{25}$ and actin polymerisation, ${ }^{26}$ respectively. Thus shape changes are likely to be a more sensitive indicator of PMN activation in vivo than are increases in the surface expression of CR1 and CR3. Interestingly, in this subgroup of patients with RA there were correlations between serum $C D$ activity and CRl expression on PMNs, between CR3 expression and PMN shape, and between levels of $C D$ in serum samples and disease activity, the last of these having been noted previously. ${ }^{17}$

Why PMNs showing morphological signs of activation should occur in the circulating leucocyte pool in patients with RA is, at first, puzzling. However, neutrophil dynamics in experimental models of acute C5a induced neutropenia ${ }^{6}$ may differ substantially from those in chronic inflammatory conditions and it is possible that the rate of margination and extravasation at inflammatory foci in patients with RA is limiting. The polarised PMNs found in blood samples from patients with RA may represent cells responding to chemotaxins but whose adherence receptors (principally CR3 or $\mathrm{CD} 11 \mathrm{~b} / \mathrm{CD} 18$ ) have not been upregulated (for the reasons discussed above), or cells whose CR3 expression and adhesiveness have been increased slightly but which have as yet failed to marginate. It has been found that gp100 ${ }^{\mathrm{MEL}} 14$, a murine adhesion protein involved in neutrophil extravasation during inflammation, and its human homologue, LAM.1, are rapidly downregulated after activation by chemotactic factors in contrast with CR3 (CD11b/CD18), which is rapidly upregulated. ${ }^{27} 28$ In addition, activated neutrophils with high CD1lb/CD18: low gp100 10 EL 14 expression are unable to localise to inflamed tissues if injected intravenously. ${ }^{29}$ Unfortunately, we were unable simultaneously to determine the expression of LAM.1.

As the number of patients with RA who were receiving a particular drug or combination of drugs was small (between one and eight), only limited conclusions on the effect of the drugs can be drawn. Nevertheless, it seems hardly surprising that higher proportions of polarised (or activated) PMNs were found in patients with $\mathrm{RA}$ receiving no drugs or receiving only NSAIDs than were found in patients receiving disease modifying drugs in addition to NSAIDs.

Our results suggest that substantial numbers of PMNs showing morphological signs of activation were circulating in the blood of patients with RA, but that the proportions of these cells found in the circulating pool were unrelated to clinical assessments of inflammatory disease activity. Significant relationships were found, however, between markers of PMN activation (i.e. PMN morphology and the surface expression of CR1 and CR3) and neutrophil lysis (i.e. serum CD activity). Further studies are clearly needed to define better the dynamics of PMN behaviour in chronic inflammatory conditions such as RA.

We are grateful to Dr J Deeks for statistical advice and to Dr Y-C Liu for assistance with some of the experiments. We thank the patients and controls who donated blood, Sister F Pegley for monitoring disease activity and collecting blood samples and the staff of Hastings Ward (London Hospital, Mile End) and of the staff of Hastings Ward (London Hospital, Mile End) and of the
Queen Mary and Westfield medical centre for collecting blood camples. Mrs R. Graham is thanked for typing this manuscipt.

1 Weissman G. Activation of neutrophils and the lesions of rheumatoid arthritis. F Lab Clin Med 1982; 100: 322-33. 2 Brown K A. The polymorphonuclear cell in rheumatoid arthritis. $\mathrm{Br} \mathcal{F}$ Rheumatol 1988; 27: 150-55.

3 Brown K A, Perry J D, Black C, Dumonde D C. Identification by cell electrophoresis of a subpopulation of polymorphonuclear cells which is increased in patients with rheumatoid arthritis and certain other rheumatological disorders. Ann Rheum Dis 1988; 47: 353-58.

4 McCarthy D A, Holburn C M, Pell B K, et al. Scanning microscopy of rheumatoid arthritis peripheral blood polymorphonuclear leucocytes. Ann Rheum Dis 1986; 45: 899-910.

5 Harlan J M. Leukocyte-endothelial interactions. Blood 1985; 65: $513-25$.

6 Fehr J, Jacob H S. In vitro granulocyte adherence and in vivo margination: two associated complement-dependent functions. Studies based on the acute neutropenia of filtration leukophoresis. F Exp Med 1977; 146: 641-52.

7 Brown K A, McCarthy D A, Perry J D, Dumonde D C. Reduction of the surface charge of blood polymorphonuclear cells by rheumatoid sera and heat induced aggregated human IgG(HAGG). Ann Rheum Dis 1988; 47: 359-63.

8 Haston W S, Shields J M. Neutrophil leucocyte chemotaxis: a simplified assay for measuring polarising responses to chemotactic factors. 7 Immunol Methods $1985 ; 81: 229-38$.

9 McCarthy D, Bernhagen J, Liu Y-C, Perry J D. A rapid preparation technique for leucocytes. $f$ Microsc 1990; 158: 63-72.

10 Barnstaple C J, Bodmer W F, Brown G, et al. Production of monoclonal antibodies to group A erythrocytes, HLA and other human cell surface antigens: new tools for genetic analysis. Cell 1978; 19: 9-20.

11 Pizzolo G, Sloane J, Beverley P, et al. Differential diagnosis of malignant lymphoma and nonlymphoid tumours using monoclonal anti-leucocyte antibody. Cancer 1980; 46: 2640-7.

12 Hogg N, MacDonald S, Slusarenko M, Beverley P C L. Monoclonal antibodies specific for human monocytes, granulocytes and endothelium. Immunology 1984; 53: 753-67.

13 Beverley P C C, Callard R E. Distinctive functional characteristics of human ' $T$ ' lymphocytes defined by $E$ rosetting of a monoclonal anti-T cell antibody. Eur 7 Immunol 1981; 11: 329-34.

14 Hildreth J E K, Gotch F M, Hidreth P D K, McMichael A J. A human lymphocyte associated antigen involved in cellmediated lympholysis. Eur f Immunol 1983; 13: 202-8. 
15 Malhotra V, Hogg N, Sim R B. Ligand binding by the p15, 95 antigen of U937 monocytic cells; properties in common with complement receptor type 3 (CR3). Eur 7 Immunol 1986; 16: 1117-23.

16 Hogg N, Ross G D, Jones D B, Slusarenko M, Walport M J, Lachmann $P$ J. Identification of an anti-monocyte monoclonal antibody that is specific for membrane complement receptor type one (CR1). Eur $\mathcal{F}$ Immunol 1984; 14: 236-40.

receptor type one (CR1). Eur F Immunol 1984; 14: 236-40.
17 Thompson P W, Jones D D, Currey H L F. Cytidine deaminase activity as a measure of acute inflammation in rheumatoid arthritis. Ann Rheum Dis 1986; 45: 9-14.

18 Romeo D. Transmembrane signalling and modulation of neutrophil behaviour. Trends Biochem $S c i$ 1982; 7: 408-13.

19 Lew D P. Receptor signalling and intracellular calcium in neutrophil activation. Eur $\mathcal{F}$ Clin Invest 1989; 19: 338-46.

20 Makinde V A, Senaldi G, Jawad A S M, Berry H, Vergani D. Reflection of disease activity in rheumatoid arthritis by indices of activation of the classical complement pathway. Ann Rheum Dis 1989; 48: 302-6.

21 Hopkins S J, Meager A. Cytokines in synovial fluid II. The presence of tumor necrosis factor and interferon. Clin Exp Immunol 1988; 73: 88-92.

22 Fontana A, Hentgartner $\mathbf{H}$, Weber E, Fehr K, Grob P J, Cohen $G$. Interleukin-1 activity in synovial fluid of patients with rheumatoid arthritis. Rheumatol Int 1982; 2: 49-53.

23 Fearon D T, Collins L A. Increased expression of C3b receptors on polymorphonuclear leucocytes induced by chemotactic factors and by purification procedures. F Immunol 1983; 130: 370-5.

24 O'Shea J J, Brown E J, Seligmann B E, Metcalf J A, Frank $M$ M, Gallen J I. Evidence for distinct intracellular pools of receptors for $\mathrm{C} 3 \mathrm{~b}$ and $\mathrm{C} 3 \mathrm{bi}$ in human neutrophils. f Immunol 1985; 134: 2580-7.

25 Showell $\mathrm{H}$ J, Freer R J, Zigmond S $\mathrm{H}$, et al. The structure activity relations of synthetic peptides as chemotactic factors and inducers of lysosomal enzyme secretion for neutrophils. and inducers of lysosomal enzym

26 Howard T H, Oresajo C O. The kinetics of chemotactic peptide-induced change in F-actin content, F-actin distribution, and the shape of neutrophils. $\mathcal{F}$ Cell Biol 1985; 101: 1078-85.

27 Kishimoto T K, Jutila M A, Berg E L, Butcher E C. Neutrophil Mac-1 and MEL-14 adhesion proteins inversely regulated by chemotactic factors. Science 1989; 245: $1238-41$.

28 Griffin J D, Spertini O, Ernst T J, et al. Granulocytemacrophage colony-stimulating factor and other cytokines regulate surface expression of the leucocyte adhesion molecule-1 on human neutrophils, monocytes and their molecule-l on human neutrophils, mon
precursors. F Immunol 1990; 145: 576-84.

29 Jutila M A, Rott L, Berg E L, Butcher E C. Function and regulation of the neutrophil MEL-14 antigen in vivo: comparison with LFA-1 and Mac-1. F Immunol 1989; 143: 3318-24. 